\title{
Appendix for Validating Migration Responses to Flooding Using Satellite and Vital Registration Data
}

By Joyce J. Chen, Valerie Mueller, Yuanyuan Jia, Steven Kuo-Hsin Tseng 

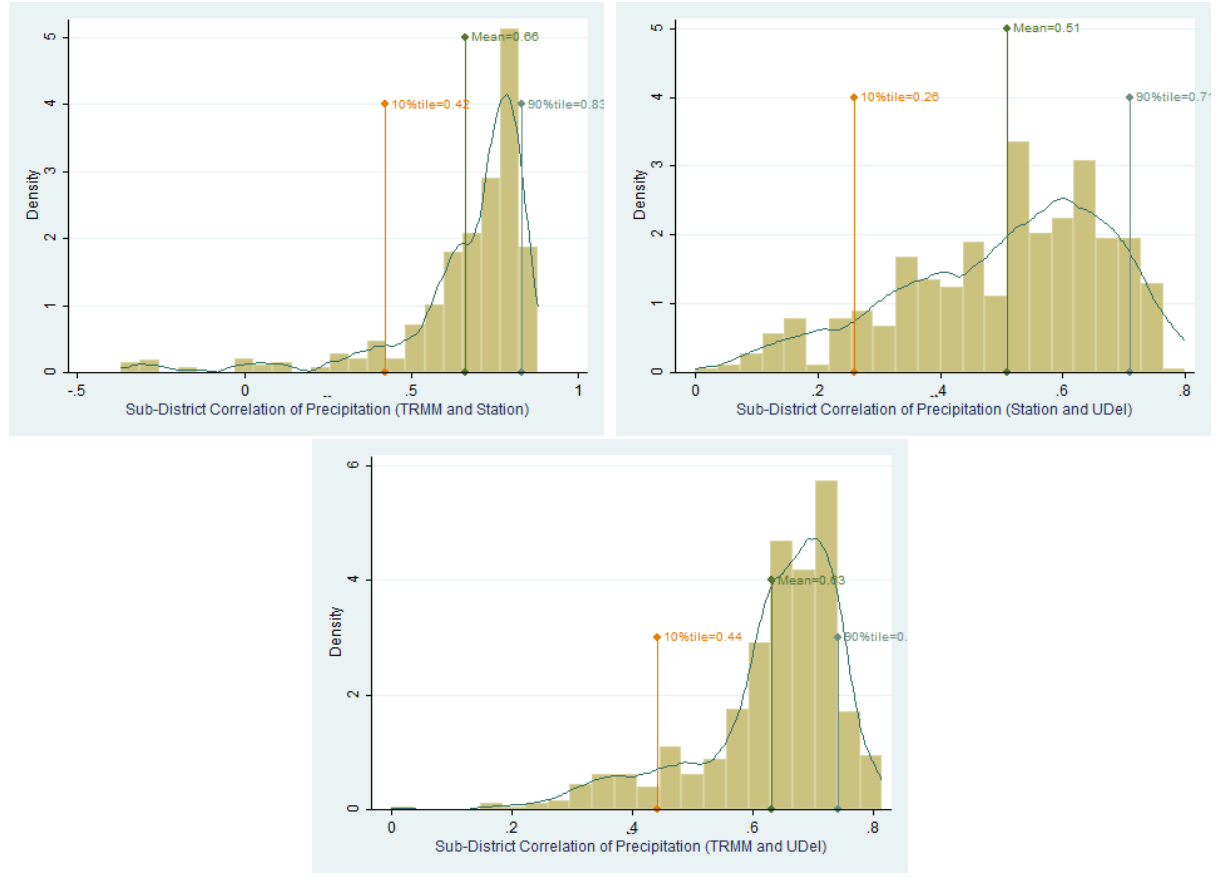

Figure 1. Epanechnikov Kernel Densities of Correlations between Data Products
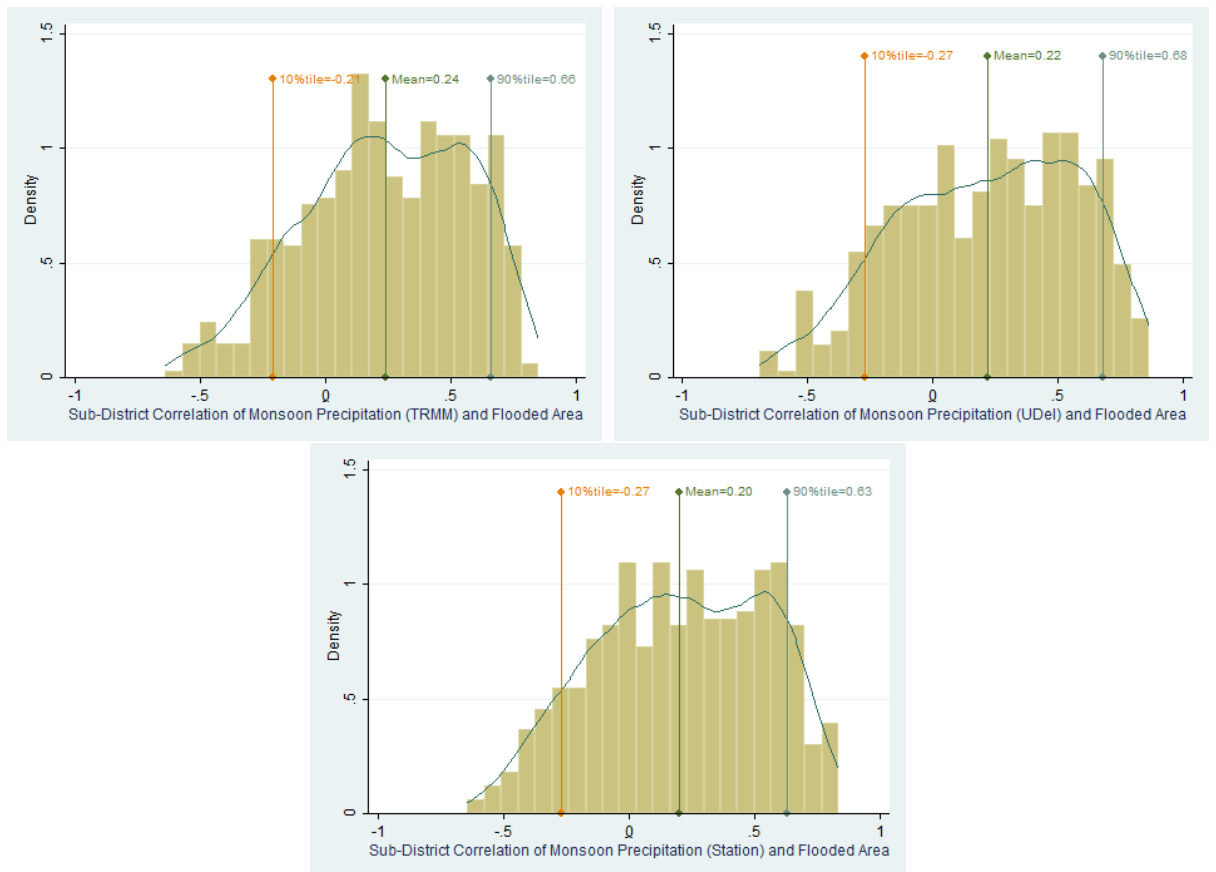

Figure 2. Epanechnikov Kernel Densities of Correlations between Monsoon Precipitation and Flood Measures 


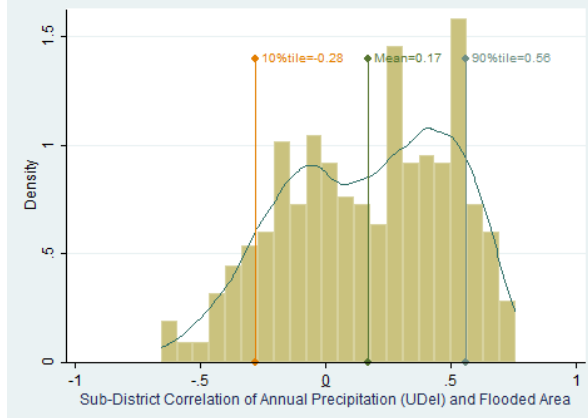

(a) All Areas

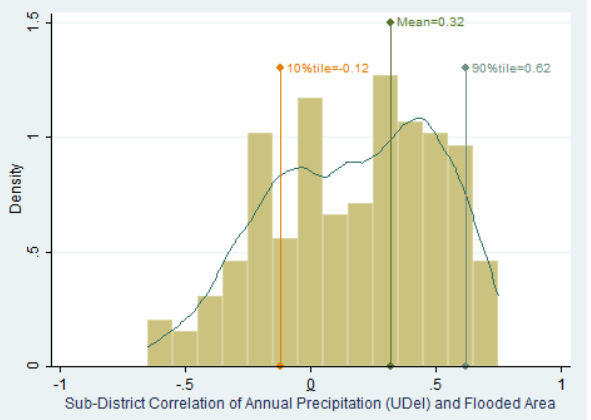

(b) High River Density (top 40\%)

Figure 3. Epanechnikov Kernel Densities of Correlations between UDel Precipitation and Flood Measures

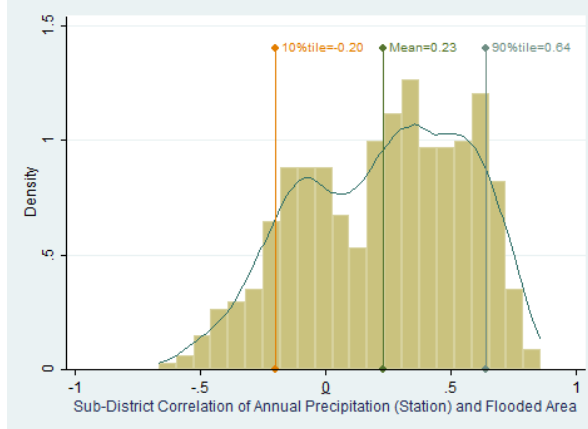

(a) All Areas

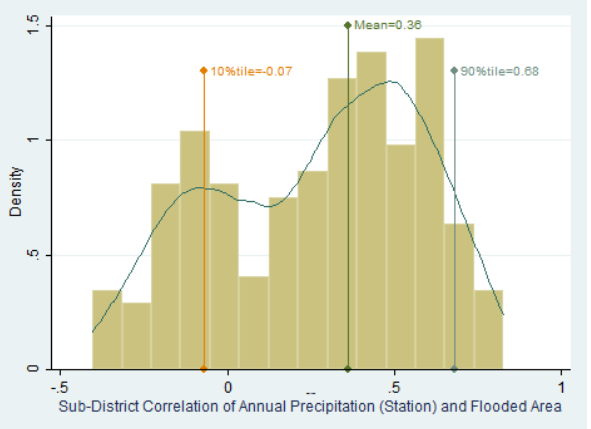

(b) High River Density (top 40\%)

Figure 4. Epanechnikov Kernel Densities of Correlations between Station Precipitation and Flood Measures 
Table 1-Summary Statistics

\begin{tabular}{|c|c|c|}
\hline & Mean & Std. \\
\hline Migration & 0.05 & 0.22 \\
\hline Annual precipitation, station & 2191.218 & 689.15 \\
\hline Annual precipitation, U. Del. & 2073.026 & 1014.53 \\
\hline Annual precipitation, TRMM & 2276.05 & 655.15 \\
\hline Flood & 0.17 & 0.18 \\
\hline Degree days & $5,874.80$ & 216.58 \\
\hline 30-year average degree days & $5,713.01$ & 151.92 \\
\hline 30-year average precipitation & $2,037.44$ & 895.62 \\
\hline River density & 0.17 & 0.096 \\
\hline Head is male & 0.89 & 0.31 \\
\hline Head's age & 45.22 & 13.76 \\
\hline Head is literate & 0.50 & 0.50 \\
\hline Head is muslim & 0.88 & 0.32 \\
\hline Head is hindu & 0.10 & 0.30 \\
\hline No. of hh members & 4.85 & 2.20 \\
\hline Male hh members $0-5$ years old & 0.31 & 0.56 \\
\hline Male hh members $6-16$ years old & 0.65 & 0.84 \\
\hline Male hh members $17-54$ years old & 1.25 & 0.87 \\
\hline Male hh members greater than 54 years old & 0.23 & 0.43 \\
\hline Female hh members $0-5$ years old & 0.30 & 0.56 \\
\hline Female hh members $6-16$ years old & 0.61 & 0.83 \\
\hline Female hh members $17-54$ years old & 1.29 & 0.76 \\
\hline Female hh members greater than 54 years old & 0.20 & 0.41 \\
\hline Primary water source comes from tap & 0.08 & 0.28 \\
\hline Primary water source comes from well & 0.90 & 0.30 \\
\hline Secondary water source comes from tap & 0.09 & 0.28 \\
\hline Secondary water source comes from well & 0.48 & 0.50 \\
\hline Has own water source & 0.53 & 0.50 \\
\hline Has kerosene as source of light & 0.46 & 0.50 \\
\hline Has electricity as source of light & 0.53 & 0.50 \\
\hline Has kerosene as source of fuel & 0.004 & 0.07 \\
\hline Has electricity as source of fuel & 0.006 & 0.08 \\
\hline Has gas as source of fuel & 0.09 & 0.29 \\
\hline Has modern or sanitary latrine & 0.58 & 0.49 \\
\hline
\end{tabular}

Note: $\mathrm{N}=1,931,954$. U. Del.=University of Delaware.

Table 2-Out-Migration Rates

\begin{tabular}{lccc}
\hline & Rural destination & Urban destination & Abroad \\
\hline & & & \\
Rural origin & 33.99 & 11.61 & 9.39 \\
Urban origin & 13.12 & 28.85 & 3.03 \\
\hline
\end{tabular}

